\title{
Upregulation of micro-ribonucleic acid- 128 cooperating with downregulation of PTEN confers metastatic potential and unfavorable prognosis in patients with primary osteosarcoma
}

\author{
This article was published in the following Dove Press journal: \\ OncoTargets and Therapy \\ 15 September 2014 \\ Number of times this article has been viewed
}

\author{
Zheng Tian ${ }^{1, *}$ \\ Bin Guo ${ }^{2, *}$ \\ Mei Yu ${ }^{3}$ \\ Chong Wang' \\ Haoshaqiang Zhang ${ }^{4}$ \\ Qingfu Liang' \\ Kunli Jiang' \\ Li Cao ${ }^{5}$ \\ 'Bone Neoplasms Department, \\ First Affiliated Hospital of Xinjiang \\ Medical University, ${ }^{2}$ Orthopedics \\ Department, Central Hospital, \\ ${ }^{3}$ Ophthalmology Department, First \\ Affiliated Hospital of Xinjiang Medical \\ University, ${ }^{4}$ Orthopedics Department, \\ People's Hospital of Xinjiang Uygur \\ Autonomous Region, ${ }^{5}$ Department of \\ Joint Surgery, First Affiliated Hospital \\ of Xinjiang Medical University, \\ Urumqi, People's Republic of China \\ *These authors contributed equally to \\ this work
}

Correspondence: Li Cao

Department of Joint Surgery, First Affiliated Hospital of Xinjiang Medical University, 137 Liyushan South Road, Urumqi, Xinjiang 830054,

People's Republic of China

Tel +86 99| 4363406

Fax +86 99। 4363406

Email tz4363406@I26.com
Objective: Abnormal expression of micro-ribonucleic acid (miRNA [miR])-128 has been observed in various human cancer types, and its validated target genes are implicated in cancerrelated cellular processes, such as cell proliferation, differentiation, and apoptosis. Especially, it has been demonstrated that miR-128 may play an important role in the proliferation of human osteosarcoma cells in vitro by directly inhibiting PTEN, which functions as a tumor suppressor in this malignancy. In the current study, we investigated the involvement of miR-128 and its target gene PTEN in tumor progression and prognosis in patients with primary osteosarcoma. Materials and methods: Expression levels of miR-128 and PTEN messenger RNA in osteosarcoma and noncancerous bone tissues obtained from 100 patients with primary osteosarcoma were detected by quantitative real-time polymerase chain reaction.

Results: Expression levels of miR-128 and PTEN messenger RNA in osteosarcoma tissues were significantly higher and lower, respectively, than those in noncancerous bone tissues (both $P<0.001$ ). In addition, high miR-128 expression and low PTEN expression, alone (miR-128high or PTEN-low) or combined (miR-128-high/PTEN-low), were all dramatically associated with poor response to chemotherapy and positive metastasis. More importantly, the associations of miR-128-high/PTEN-low expression with these clinicopathological parameters were more significant than miR-128-high or PTEN-low alone. Finally, miR-128 expression, PTEN expression, miR-128/PTEN expression, the response to chemotherapy and the metastatic status were all identified as independent prognostic factors for overall survival and disease-free survival.

Conclusion: These findings indicate for the first time that the deregulation of miR-128 and its target gene PTEN may be involved in the aggressive progression of human osteosarcoma. Notably, the upregulation of miR-128 cooperating with the downregulation of PTEN may confer an unfavorable prognosis in patients with this malignancy.

Keywords: osteosarcoma, microRNA-128, phosphatase and tensin homolog, clinicopathological parameter, prognosis

\section{Introduction}

As the most common type of primary bone tumor, osteosarcoma predominantly occurs around regions with active bone growth and repair. ${ }^{1}$ This malignant mesenchymal neoplasm mainly occurs in adolescents and young adults, and directly produces defective osteoid. $^{2}$ Osteosarcoma has a highly malignant tendency to rapidly destroy the surrounding tissues and to metastasize. Despite the considerable increase in the survival rate of patients with osteosarcoma to $65 \%-75 \%$, due to great advances in surgical 
technology and combined therapeutic strategies, the survival of patients with pulmonary metastases and advanced clinical stage remains poor. ${ }^{3}$ Therefore, accumulating attempts have been made to identify the prognostic factors for survival of patients with osteosarcomas and to identify patients with a high risk of developing recurrence or metastases from those with a low risk. ${ }^{4}$ Several clinicopathological characteristics, such as tumor size, tumor grade, response to chemotherapy, and status of metastasis, have been used as prognostic factors for patients with osteosarcomas, ${ }^{5}$ but their sensitivities and specificities are not satisfactory, implying that several genetic or molecular events may be implicated in the tumorigenesis of this malignancy.

Malignant transformation, in which cells change their identities, has been demonstrated to be mediated by epigenetic mechanisms, including deoxyribonucleic acid methylation, histone modifications, and micro-ribonucleic acids (miRNAs). ${ }^{6}$ As a class of small noncoding and endogenous regulatory RNA molecules usually 19-25 nucleotides in length, miRNAs can regulate gene expression via specific sites at the 3 '-untranslated region of target messenger RNAs (mRNAs), causing translational repression or degradation. ${ }^{7}$ To date, hundreds of miRNAs have been found both in prokaryotes and eukaryotes. Among them, there are more than 1,500 human miRNAs, each of which is predicted to have the potential to target dozens of genes. ${ }^{8}$ The partial complementarity of the miRNA/mRNA duplex allows for miRNAs to target multiple mRNAs of genes that may share in the regulation of common cellular processes, such as hematopoietic cell differentiation, neural development, cell cycle, apoptosis, and metabolism. ${ }^{9}$ An increasing number of studies have demonstrated a crucial role for miRNAs in tumorigenesis. They function as tumor suppressors or oncogenes by regulating the expression of their target genes. Since the distinct patterns of miRNA expression have been observed in many cancers, several research groups have utilized miRNA-expression profiles as diagnostic and prognostic markers in various human malignancies, including osteosarcoma. ${ }^{10,11}$

miR-128, a brain-enriched miRNA, has been demonstrated to have tissue-specific and development-specific expression patterns, mainly in neurons. ${ }^{12}$ Its abnormal expression has been detected not only in tissues but also in the blood of some malignant tumors, implying its involvement in tumorigenesis. ${ }^{13}$ Aberrant expression of miR-128 may contribute to the malignant phenotypes of cancer cells, such as proliferation, cell motility, invasion, apoptosis, and self-renewal. For example, miR-128 was significantly upregulated in patients with acute lymphoblastic leukemia. ${ }^{14}$ A subsequent study supported that it had diagnostic value in acute leukemia. ${ }^{15}$ Shen et al $^{16}$ reported that miR-128 expression was significantly increased in osteosarcoma tissues compared to adjacent normal tissues. Ectopic overexpression of miR-128 significantly promoted the proliferation of osteosarcoma cells, while suppression of miR-128 by its antisense inhibited the proliferation of osteosarcoma cells. In contrast, a reduced level of miR-128 was first identified in glioblastoma. ${ }^{17}$ It inhibits tumor-cell proliferation in glioma, neuroblastoma, colorectal cancer, and prostate cancer by cell-cycle arrest. ${ }^{18-20}$ Reduced miR-128 in breast tumorinitiating cells may induce chemotherapeutic resistance. ${ }^{21}$ These findings suggest that this miRNA may function either as an oncogene or a tumor suppressor, depending on different cancer types.

Of note, it has been indicated that PTEN, which is a well-established tumor suppressor, is a direct target of miR-128 in osteosarcoma cells. miR-128/PTEN signaling may be an important pathway regulating osteosarcoma-cell proliferation. ${ }^{16}$ However, the clinical significance of the deregulation of the miR-128/PTEN axis in human osteosarcoma has not been fully elucidated. To address this problem, the aim of the present study was to determine the involvement of miR-128 and its target gene PTEN in tumor progression and prognosis in patients with primary osteosarcoma.

\section{Materials and methods \\ Patients and tissue samples}

One hundred patients with primary osteosarcoma who received surgical treatment at the First Affiliated Hospital of Xinjiang Medical University (Xinjiang Uygur Autonomous Region, People's Republic of China) and the People's Hospital of Xinjiang Uygur Autonomous Region between 2005 and 2009 were enrolled in the current study. One hundred pairs of osteosarcoma-tissue samples and corresponding noncancerous bone-tissue samples were collected from the same patients with osteosarcomas. All the samples were biopsy materials before any therapy, and were frozen and stored at $-80^{\circ} \mathrm{C}$ for RNA extraction. This study was approved by the Research Ethics Committee of the First Affiliated Hospital of Xinjiang Medical University and the People's Hospital of Xinjiang Uygur Autonomous Region. Written informed consent was obtained from all of the patients.

The patients ranged from 8 to 68 years of age (median 18 years, mean 22.68 years). The study population was 68 males and 32 females. After establishing 
the diagnosis, all patients were treated with the same neoadjuvant chemotherapy consisting of methotrexate, doxorubicin, cisplatin, and ifosfamide. All drugs were given intravenously. Following neoadjuvant chemotherapy, the patients underwent wide resection of tumors. Response to chemotherapy was classified as poor ( $<90 \%$ tumor necrosis) and good ( $>90 \%$ tumor necrosis) through histologic analysis of tumor specimens after surgery. ${ }^{22}$ The clinicopathologic data were retrospectively reviewed and are summarized in Table 1.

All 100 patients with primary osteosarcomas received follow-up and were monitored with computed tomography performed every 3 months during the first 3 years after chemotherapy, every 4 months during years 4 and 5, and every 6 months thereafter. The development of local recurrence and distant metastasis were detected by computed tomography scans or magnetic resonance imaging. The median follow-up was 29.82 months (range 5.26-38.89 months). For survival analysis, overall survival (OS) was defined as the time interval from the date of diagnosis at our center to the date of death or the last follow-up. Disease-free survival (DFS) was defined as the time interval from diagnosis at our center to progressive disease, death of any other cause than progression, or a second primary cancer. The 3 -year OS was 75\%, and the 3 -year DFS was $58 \%$ for all patients.

\section{RNA extraction}

Total RNA and small RNA were extracted from fresh osteosarcoma and corresponding noncancerous tissues with an RNeasy minikit (Qiagen, Venlo, the Netherlands) and a mirVana ${ }^{\mathrm{TM}}$ miRNA isolation kit (Thermo Fisher Scientific, Waltham, MA, USA), respectively, according to the manufacturer's instructions. Concentration and purity of the RNA samples were assessed using the NanoDrop ${ }^{\circledR}$ 1000 Spectrophotometer (Thermo Fisher Scientific). To estimate concentration, 1 unit of $\mathrm{OD}_{260}$ equals $40 \mu \mathrm{g} / \mathrm{mL}$ RNA. For purity, the 260:280 ratio should be above 1.8 .

Table I Association of micro-ribonucleic acid (miR)-I28 and PTEN expression with clinicopathological features of pediatric osteosarcoma

\begin{tabular}{|c|c|c|c|c|c|c|c|}
\hline $\begin{array}{l}\text { Clinicopathological } \\
\text { features }\end{array}$ & $\begin{array}{l}\text { Cases, } \\
\mathrm{n}(\%)\end{array}$ & $\begin{array}{l}\text { miR-I 28- } \\
\text { high, n (\%) }\end{array}$ & $P$ & $\begin{array}{l}\text { PTEN-low, } \\
\text { n (\%) }\end{array}$ & $P$ & $\begin{array}{l}\text { miR-I 28-high/ } \\
\text { PTEN-low, n (\%) }\end{array}$ & $P$ \\
\hline \multicolumn{8}{|l|}{ Age (years) } \\
\hline$<18$ & $40(40)$ & $20(50)$ & NS & $22(55)$ & NS & $15(37.50)$ & NS \\
\hline$\geq 18$ & $60(60)$ & $32(53.33)$ & & $34(56.67)$ & & $25(4 I .67)$ & \\
\hline \multicolumn{8}{|l|}{ Sex } \\
\hline Male & $68(68)$ & $38(55.88)$ & NS & $40(58.82)$ & NS & $25(36.76)$ & NS \\
\hline Female & $32(32)$ & $14(43.75)$ & & $16(50)$ & & $15(46.88)$ & \\
\hline \multicolumn{8}{|l|}{ Tumor site } \\
\hline Femur & $55(55)$ & $28(50.91)$ & NS & $28(50.91)$ & NS & $20(36.36)$ & NS \\
\hline Tibia & $20(20)$ & $10(50)$ & & $10(50)$ & & $8(40)$ & \\
\hline Humeral bone & $15(15)$ & $8(53.33)$ & & $8(53.33)$ & & $8(53.33)$ & \\
\hline Other & $10(10)$ & $6(60)$ & & $6(60)$ & & $4(40)$ & \\
\hline \multicolumn{8}{|l|}{ Histologic type } \\
\hline Osteoblastic & $55(55)$ & 28 (50.9l) & NS & $28(50.91)$ & NS & $20(36.36)$ & NS \\
\hline Chondroblastic & $20(20)$ & $10(50)$ & & $10(50)$ & & $8(40)$ & \\
\hline Fibroblastic & $15(15)$ & $8(53.33)$ & & $8(53.33)$ & & $8(53.33)$ & \\
\hline Telangiectatic & $10(10)$ & $6(60)$ & & $6(60)$ & & $4(40)$ & \\
\hline \multicolumn{8}{|l|}{ Tumor grade } \\
\hline Low & $15(15)$ & 7 (46.67) & NS & $9(60)$ & NS & 7 (46.67) & NS \\
\hline High & $85(85)$ & $45(52.94)$ & & 47 (55.29) & & $33(38.82)$ & \\
\hline \multicolumn{8}{|l|}{ Metastasis } \\
\hline Absent & $60(60)$ & $22(36.67)$ & $<0.001$ & $23(38.33)$ & $<0.001$ & $10(16.67)$ & $<0.001$ \\
\hline Present & $40(40)$ & $30(75)$ & & $33(82.50)$ & & $30(75)$ & \\
\hline \multicolumn{8}{|l|}{ Recurrence } \\
\hline Absent & $70(60)$ & 37 (52.86) & NS & $38(54.29)$ & NS & $25(35.7 I)$ & NS \\
\hline Present & $30(40)$ & $15(50)$ & & $18(60)$ & & $15(50)$ & \\
\hline \multicolumn{8}{|c|}{ Response to preoperative chemotherapy } \\
\hline Good & $50(50)$ & $20(40)$ & 0.01 & $20(40)$ & 0.01 & $10(20)$ & 0.006 \\
\hline Poor & $50(50)$ & $32(64)$ & & $36(72)$ & & $30(60)$ & \\
\hline
\end{tabular}

Abbreviation: NS, not significant. 


\section{Quantitative real-time reverse- transcriptase polymerase chain reaction for miR- 128}

Reverse transcription was performed using the TaqMan ${ }^{\circledR}$ miRNA reverse-transcription kit (Thermo Fisher Scientific) according to the manufacturer's instructions. RNU6 (Thermo Fisher Scientific) was used as the endogenous control for the expression of miR-128. Real-time PCR (RT-PCR) reactions for miRNAs were performed in triplicate in $20 \mu \mathrm{L}$ volumes. The sequences of the primers were as follows: miR-128 forward, 5'-CGC GCT CAC AGT GAA CCG-3'; reverse, 5'-GTG CAG GGT CCG AGG T-3'; U6 forward 5'-GCG CGT CGT GAA GCG TTC- $3^{\prime}$ and reverse $5^{\prime}$-GTG CAG GGT CCG AGG T-3'. Quantitative miRNA-expression data were acquired and analyzed using a 7500 RT-PCR system (Thermo Fisher Scientific). The quantitative RT-PCR (qRT-PCR) assays for a particular gene were undertaken at the same time for all samples under identical conditions, in triplicate. Relative expression levels of miR-128 were calculated using the $2^{-\Delta \Delta C t}$ relative quantification method. ${ }^{23}$

\section{qRT-PCR for PTEN}

Reverse transcription was performed using a ReverTra Ace $^{\circledR}$ qRT kit (Toyobo, Osaka, Japan) according to the manufacturer's instructions. Expression level of PTEN mRNA was quantitatively assayed by RT-PCR using TaqMan geneexpression assays. The $\beta$-actin was used as the endogenous control. The sequences of the primers were as follows: human PTEN forward 5'-CCGAAA GGT TTT GCT ACC ATT CT-3' and reverse $5^{\prime}$-AAA ATTA TTT CCT TTC TGA GCA TTC C-3'; human $\beta$-actin forward 5'-CCT GTA CGC CAA CAC AGT GC- $3^{\prime}$ and reverse $5^{\prime}$-ATA CTC CTG CTT GCT GAT CC-3'. Quantitative mRNA-expression data were acquired and analyzed by an the 7500 RT-PCR system. The qRT-PCR assays for a particular gene were undertaken at the same time for all samples under identical conditions, in triplicate. Relative expression levels of PTEN mRNA were calculated using the $2^{-\Delta \Delta C t}$ relative quantification method. ${ }^{23}$

\section{Statistical analysis}

The SPSS statistical package (version 17.0; SPSS, Chicago, IL, USA) was used for statistical analysis. Continuous variables were expressed as means \pm standard deviation. The paired $t$-test was used to evaluate differences of miR-128- or PTEN-expression levels in osteosarcoma and corresponding noncancerous bone tissues. Clinicopathological parameters according to miR-128 and PTEN expression, alone (miR-128-high or PTEN-low) or combined (miR-128-high/ PTEN-low), were compared by $\chi^{2}$ tests. The Kaplan-Meier test was used to determine the probability of survival, and data were analyzed by the log-rank test. Differences were considered statistically significant when $P<0.05$.

\section{Results \\ Upregulation of miR- 128 and downregulation of PTEN mRNA in human osteosarcoma tissues}

As shown in Figure 1, the expression levels of miR-128 (tumor versus normal, $4.61 \pm 0.87$ versus $2.59 \pm 0.67 ; P<0.001$ ) and PTEN mRNA (tumor versus normal, $2.99 \pm 1.29$ versus $4.83 \pm 1.607 ; P<0.001$ ) were respectively higher and lower in osteosarcoma tissues than those in noncancerous bone tissues significantly. In addition, the median values of miR-128 (4.56) and PTEN mRNA (3.10) expression levels in all osteosarcoma tissues were used as cutoff points to classify 100 patients with osteosarcomas into miR-128-low ( $\mathrm{n}=48$ ), miR-128-high $(\mathrm{n}=52)$, PTEN-low $(\mathrm{n}=56)$, and PTEN-high $(\mathrm{n}=44)$ expression groups. On this basis, $16(16 \%)$ cases showed low expression of both miR-128 and PTEN, 12 (12.00\%) cases showed high expression of both miR-128 and PTEN, 40 (40\%) cases showed miR-128-high and PTEN-low expression, and 32 (32\%) cases showed miR-128-low and PTEN-high expression. Notably, the downregulation of miR-128 was negatively correlated with the upregulation of PTEN mRNA in osteosarcoma tissues (Spearman's correlation, $r=-0.62 ; P=0.01$; Figure $1 \mathrm{C}$ ).

\section{miR-I 28 upregulation and PTEN downregulation, alone or combined, \\ are associated with aggressive clinicopathological features of human osteosarcoma}

As shown in Table 1, high miR-128 expression and low PTEN expression, alone (miR-128-high or PTEN-low) or combined (miR-128-high/PTEN-low), were all dramatically associated with poor response to chemotherapy $(P=0.01,0.01$, and 0.006 , respectively) and positive metastasis (all $P<0.001$ ). More importantly, the associations of miR-128-high/PTEN-low expression with these clinicopathological parameters were more significant than miR-128-high or PTEN-low alone.

\section{miR-I 28 upregulation and PTEN downregulation, alone or combined, confer poor prognosis in human osteosarcoma}

Using the Kaplan-Meier method and log-rank test, both the OS and DFS of osteosarcoma patients with high miR-128 expression (Figure 2A and D, respectively; both $P<0.001$ ) 

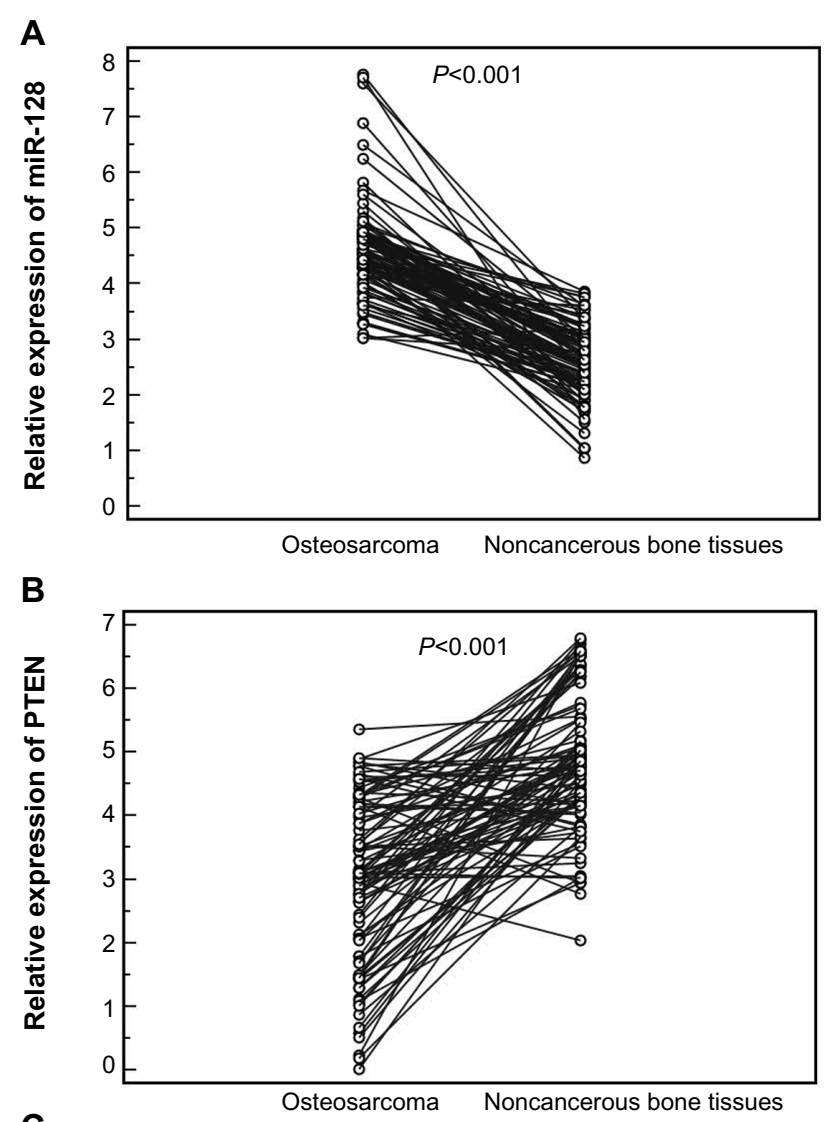

C

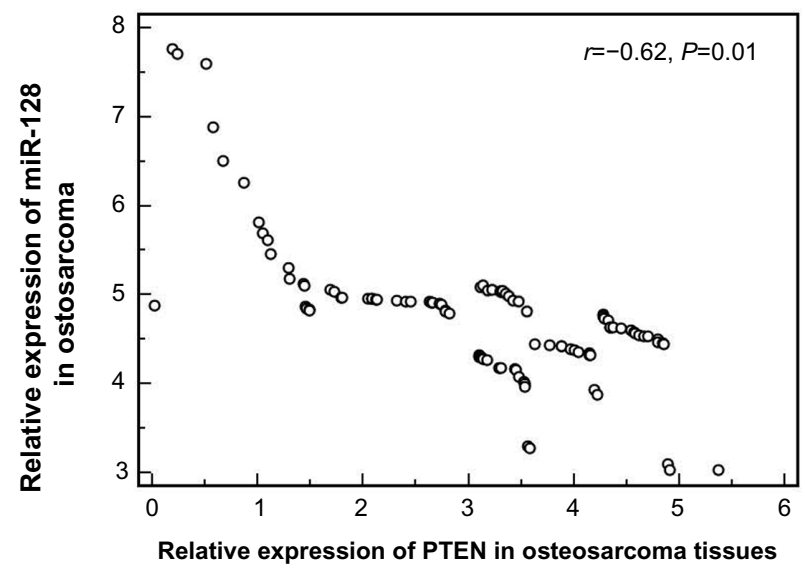

Figure I Expression levels of micro-ribonucleic acid (miRNA [miR])-128 (A) and PTEN messenger RNA (mRNA) (B) in 100 pairs of osteosarcoma and corresponding noncancerous bone tissues detected by quantitative real-time polymerase chainreaction assay.

Note: The results showed that the expression levels of miR-128 and PTEN mRNA were respectively higher and lower in osteosarcoma tissues than those in noncancerous bone tissues (both $P<0.001$ ). (C) The upregulation of miR-128 was negatively correlated with the downregulation of PTEN mRNA in osteosarcoma tissues (Spearman's correlation, $r=-0.62 ; P=0.01$ ).

and low PTEN expression (Figure 2B and E, respectively; both $P<0.001$ ) were significantly shorter than those with low miR-128 expression and high PTEN expression. With regard to the combined expression of miR-128 and PTEN, the osteosarcoma patients with miR-128-high/PTEN-low expression had the shortest OS $(P<0.001$, Figure $2 \mathrm{C})$ and DFS $(P<0.001$,
Figure $2 \mathrm{~F}$ ) when compared with patients in the other three groups. The survival benefits were also found in those with low tumor grade (both $P=0.02$ ), better response to preoperative chemotherapy (both $P<0.001$ ), and without metastasis (both $P=0.01)$ and recurrence $(P=0.02)$ for OS and DFS.

A Cox proportional-hazard model confirmed that miR128 expression (OS, relative risk [RR] 7.88, 95\% confidence interval [CI] 1.10-16.49, $P=0.008$; DFS, RR 8.36, 95\% CI 1.10-18.29, $P=0.006$ ), PTEN expression (OS, RR 7.22, 95\% CI 1.06-15.39, $P=0.01$; DFS, RR 8.12, 95\% CI 1.10-17.93, $P=0.006$ ), miR-128/PTEN expression (OS, RR 16.32, 95\% CI 1.86-35.18, $P<0.001$; DFS, RR 18.99, 95\% CI 1.97$39.76, P<0.001$ ), response to preoperative chemotherapy (OS, RR 7.28, 95\% CI 1.08-15.11, $P=0.01$; DFS, RR 8.25, 95\% CI 1.11-17.99, $P=0.006$ ), and metastasis status (OS, RR 6.44, 95\% CI 0.96-13.62, $P=0.02$; DFS, RR 6.92, 95\% CI $1.00-14.06, P=0.01)$ were independent prognostic factors of unfavorable survival in human osteosarcoma (Table 2).

\section{Discussion}

Accumulating evidence has suggested that miRNAs may play crucial roles in the tumor progression and prognosis in human primary osteosarcoma via regulating the expression of their direct target genes. In the current study, there were four main findings according to our data. Firstly, miR-128 and its direct target gene PTEN were respectively upregulated and downregulated in osteosarcoma tissues compared with noncancerous bone tissues, suggesting that the aberrant expression of the two markers may be involved in the tumorigenesis of human osteosarcoma. Second, high miR-128 expression, low PTEN expression, and miR-128-high/PTEN-low combined expression were all significantly associated with the aggressive clinicopathological characteristics of osteosarcomas, including the presence of tumor metastasis and poor response to chemotherapy, suggesting that the dysregulation of the miR128/PTEN axis may be implicated in the tumor progression of human osteosarcoma. Third, the associations of miR-128high/PTEN-low combined expression with these clinicopathological parameters were more significant than miR-128-high or PTEN-low expression alone. Finally, both the univariate and multivariate analyses showed that high miR-128 expression, low PTEN expression, and miR-128-high/PTEN-low combined expression were all independent prognostic factors for patients with osteosarcomas, suggesting that dysregulation of the miR-128/PTEN axis may predict decreased survival and a greater probability of disease progression.

As a kind of intronic miRNA, miR-128 is encoded by two distinct genes - miR-128-1 and miR-128-2 - which are embedded in the introns of the R3HDMI and RCS 

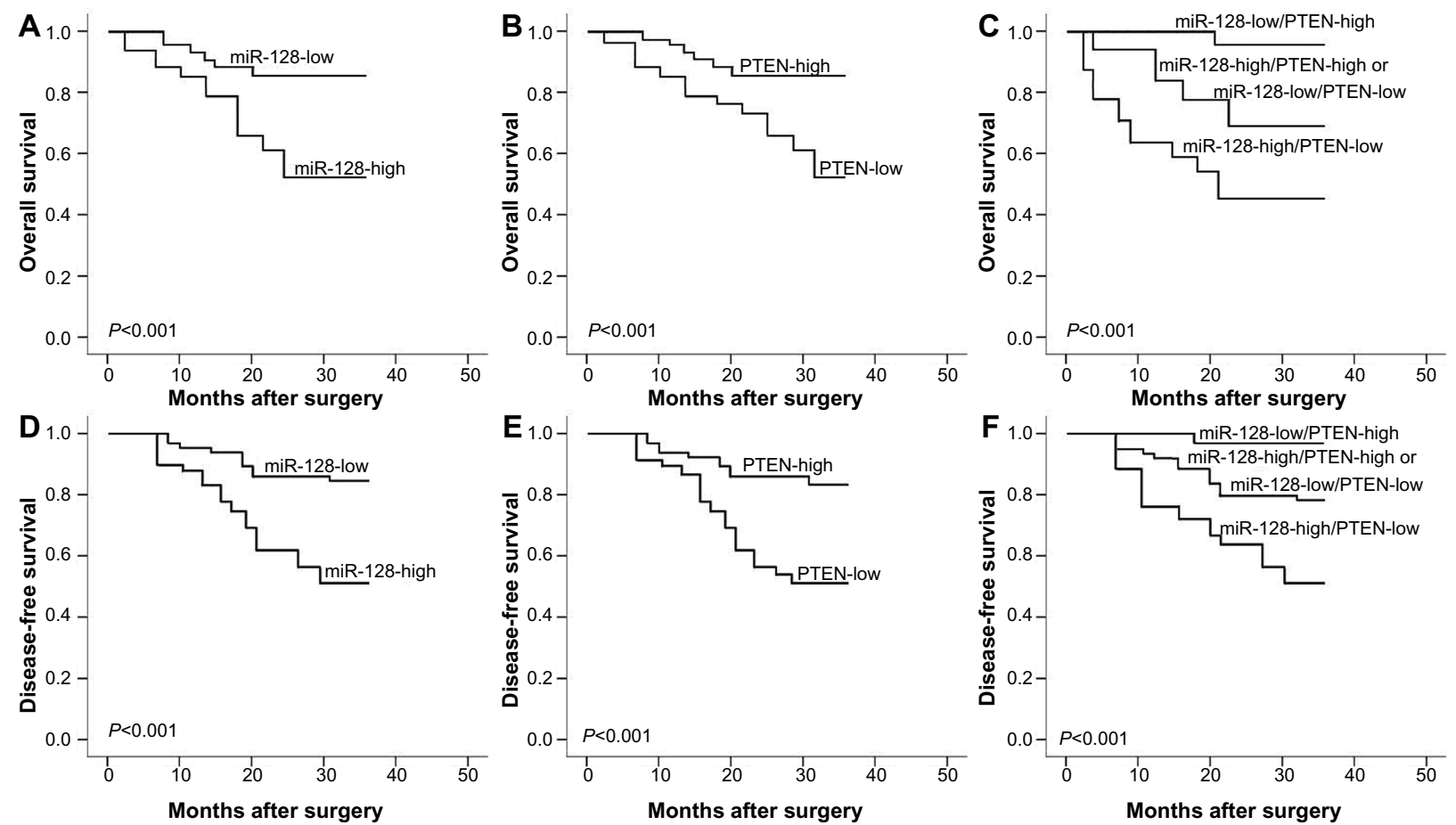

Figure 2 (A-F) Kaplan-Meier survival curves for osteosarcoma patients with micro-ribonucleic acid (miR)-I28 expression and PTEN expression, alone (miR-I28-high/low or PTEN-low/high) or combined (miR-128-high/PTEN-low).

Note: (A-C) Overall survival; (D-F) disease-free survival.

genes, located on human chromosomes 2q21.3 and 3p22.3, respectively. ${ }^{24}$ Both miR-128-1 and miR-128-2 are processed to generate the same mature miRNAs with identical sequences - miR-128 - the aberrant expression of which has been observed in many kinds of malignant tumors, including acute lymphoblastic leukemia, acute myeloid leukemia, glioma, osteosarcoma, breast cancer, lung cancer, gastric cancer, pancreatic cancer, colon cancer, prostate cancer, and ovarian cancer. ${ }^{14-21}$ It has been demonstrated that miR-128 can regulate the proliferation, differentiation, and apoptosis of various tumor cells via targeting several genes. Shi et al ${ }^{18}$ reported that miR-128 may inhibit the tumor growth and angiogenesis of gliomas by targeting p70S6K1. Takahashi et $\mathrm{al}^{19}$ found that miR-128 could inhibit colorectal cancercell proliferation by suppressing the expression of NEK2.
Zhu et $\mathrm{a}^{20}$ indicated that the reduction in miR-128 leading to Bmi-1 and ABCC5 overexpression may be a stem cell-like feature of breast tumor-initiating cells, contributing to chemotherapeutic resistance in breast cancers. Godlewski et $\mathrm{al}^{21}$ showed that miR-128 expression could significantly reduce glioma-cell proliferation in vitro and glioma-xenograft growth in vivo by decreasing the expression of the oncogene Bmi-1. Shen et $\mathrm{al}^{16}$ also found that miR-128 expression was significantly increased in osteosarcoma tissues compared to adjacent normal tissues, and ectopic overexpression of miR-128 could promote the proliferation of osteosarcoma cell lines, while suppression of miR-128 by its antisense could inhibit the proliferation of osteosarcoma cell lines, consistent with which, our data further showed the significant association of miR-128 upregulation in osteosarcoma

Table 2 Multivariate survival analysis of overall survival (OS) and disease-free survival (DFS) in 100 patients with osteosarcoma

\begin{tabular}{|c|c|c|c|c|c|c|}
\hline \multirow[t]{2}{*}{ Variables } & \multicolumn{3}{|l|}{ OS } & \multicolumn{3}{|l|}{ DFS } \\
\hline & $\mathbf{R R}$ & $95 \% \mathrm{Cl}$ & $\boldsymbol{P}$ & $\mathbf{R R}$ & $95 \% \mathrm{Cl}$ & $P$ \\
\hline miR-128 expression & 7.88 & $1.10-16.49$ & 0.008 & 8.36 & $1.10-18.29$ & 0.006 \\
\hline PTEN expression & 7.22 & $1.06-15.39$ & 0.01 & 8.12 & $1.10-17.93$ & 0.006 \\
\hline miR-I28/PTEN expression & 16.32 & $1.86-35.18$ & $<0.001$ & 18.99 & $1.97-39.76$ & $<0.001$ \\
\hline Tumor grade & 2.42 & $0.6 I-5.16$ & NS & 3.37 & $0.70-7.38$ & NS \\
\hline Response to preoperative chemotherapy & 7.28 & $1.08-15.11$ & 0.01 & 8.25 & $1.11-17.99$ & 0.006 \\
\hline Metastasis status & 6.44 & $0.96-13.62$ & 0.02 & 6.92 & $1.00-14.06$ & 0.01 \\
\hline Recurrence status & 3.26 & $0.68-7.02$ & NS & 3.59 & $0.7 I-7.83$ & NS \\
\hline
\end{tabular}

Abbreviations: miR, micro-ribonucleic acid; RR, relative risk; $\mathrm{Cl}$, confidence interval; NS, not significant. 
tissues with the presence of tumor metastasis, poor response to chemotherapy, and shorter survival after surgery.

As a well established tumor-suppressor gene, PTEN is one the most frequently mutated genes in various human cancers. ${ }^{25}$ It acts as a lipid phosphatase, dephosphorylating the $3^{\prime}$ position of phosphoinositide 3,4,5-triphosphate (PIP3), which is the product of a potent proto-oncogenic kinase phosphoinositide 3-kinase (PI3K) - and the trigger for activation of the PI3K pathway. The relevance of this pathway in cancer has been highlighted by the elevated number of components within the cascades, such as the activation of AKT kinases, which play crucial roles in cell survival, cell proliferation, angiogenesis, and anabolic metabolism, and represent one of the main targets for cancer therapy. ${ }^{26}$ In osteosarcomas, Gao et $\mathrm{al}^{27}$ and Shen et $\mathrm{al}^{16}$ both observed the downregulation of PTEN expression in osteosarcoma tissues and cell lines, which was in line with our findings. In these previous studies, PTEN functions as a direct target for miR-17 and miR-128, respectively. They found that the inhibition of PTEN expression by miR-17 and miR-128 could suppress tumor-cell proliferation, tumor growth, and metastasis in osteosarcoma cell lines. In addition to these findings of in vitro systems, our study based on clinical materials determined that the downregulation of PTEN may be significantly associated with aggressive tumor progression and poor prognosis in patients with primary osteosarcoma. More strikingly, we found that deregulation of the miR-128/ PTEN axis was more significantly correlated with advanced clinical parameters of patients with osteosarcoma, and we also determined the value of miR-128/PTEN axis in judging unfavorable prognosis in patients with osteosarcomas.

In conclusion, these findings indicate for the first time that the deregulation of miR-128 and its target gene PTEN may be involved in the aggressive progression of human osteosarcoma. Notably, the upregulation of miR-128 cooperating with the downregulation of PTEN may confer an unfavorable prognosis in patients with this malignancy.

\section{Disclosure}

The authors report no conflicts of interest in this work.

\section{References}

1. Yang J, Zhang W. New molecular insights into osteosarcoma targeted therapy. Curr Opin Oncol. 2013;25:398-406.

2. Gill J, Ahluwalia MK, Geller D, Gorlick R. New targets and approaches in osteosarcoma. Pharmacol Ther. 2013;137:89-99.

3. Osborne TS, Khanna C. A review of the association between osteosarcoma metastasis and protein translation. J Comp Pathol. 2012;146:132-142.

4. Poletajew S, Fus L, Wasiutyński A. Current concepts on pathogenesis and biology of metastatic osteosarcoma tumors. Ortop Traumatol Rehabil. 2011;13:537-545.
5. PosthumaDeBoer J, Witlox MA, Kaspers GJ, van Royen BJ. Molecular alterations as target for therapy in metastatic osteosarcoma: a review of literature. Clin Exp Metastasis. 2011;28:493-503.

6. Chou J, Shahi P, Werb Z. MicroRNA-mediated regulation of the tumor microenvironment. Cell Cycle. 2013;12:3262-3271.

7. Miao J, Wu S, Peng Z, Tania M, Zhang C. MicroRnAs in osteosarcoma: diagnostic and therapeutic aspects. Tumour Biol. 2013;34:2093-2098.

8. Zhou G, Shi X, Zhang J, Wu S, Zhao J. MicroRNAs in osteosarcoma: from biological players to clinical contributors, a review. J Int Med Res. 2013;41:1-12.

9. Liang W, Gao B, Fu P. The miRNAs in the pathgenesis of osteosarcoma. Front Biosci (Landmark Ed). 2013;18:788-794.

10. Mu Y, Zhang H, Che L, Li K. Clinical significance of microRNA-183/ Ezrin axis in judging the prognosis of patients with osteosarcoma. Med Oncol. 2014;31:821.

11. Zhao H, Li M, Li L. miR-133b is down-regulated in human osteosarcoma and inhibits osteosarcoma cells proliferation, migration and invasion, and promotes apoptosis. PLoS One. 2013;8:e83571.

12. Peruzzi P, Bronisz A, Nowicki MO. MicroRNA-128 coordinately targets polycomb repressor complexes in glioma stem cells. Neuro Oncol. 2013; 15:1212-1224.

13. Li M, Fu W, Wo L. miR-128 and its target genes in tumorigenesis and metastasis. Exp Cell Res. 2013;319:3059-3064.

14. Fulci V, Colombo T, Chiaretti S. Characterization of B- and T-lineage acute lymphoblastic leukemia by integrated analysis of microRNA and mRNA expression profiles. Genes Chromosomes Cancer. 2009;48: 1069-1082.

15. Mi S, Lu J, Sun M. MicroRNA expression signatures accurately discriminate acute lymphoblastic leukemia from acute myeloid leukemia. Proc Natl Acad Sci U S A. 2007;104:19971-19976.

16. Shen L, Chen XD, Zhang YH. MicroRNA-128 promotes proliferation in osteosarcoma cells by downregulating PTEN. Tumour Biol. 2014;35: 2069-2074.

17. Ciafrè SA, Galardi S, Mangiola A. Extensive modulation of a set of microRNAs in primary glioblastoma. Biochem Biophys Res Commun. 2005;334:1351-1358.

18. Shi ZM, Wang J, Yan Z. miR-128 inhibits tumor growth and angiogenesis by targeting p70S6K1. PLoS One. 2012;7:e32709.

19. Takahashi Y, Iwaya T, Sawada G. Up-regulation of NEK2 by microRNA-128 methylation is associated with poor prognosis in colorectal cancer. Ann Surg Oncol. 2014;21:205-212.

20. Zhu Y, Yu F, Jiao Y. Reduced miR-128 in breast tumor-initiating cells induces chemotherapeutic resistance via Bmi-1 and ABCC5. Clin Cancer Res. 2011;17:7105-7115.

21. Godlewski J, Nowicki MO, Bronisz A. Targeting of the Bmi-1 oncogene/stem cell renewal factor by microRNA-128 inhibits glioma proliferation and self-renewal. Cancer Res. 2008;68: 9125-9130.

22. Bacci G, Bertoni F, Longhi A. Neoadjuvant chemotherapy for highgrade central osteosarcoma of the extremity. Histologic response to preoperative chemotherapy correlates with histologic subtype of the tumor. Cancer. 2003;97:3068-3075.

23. Earle JS, Luthra R, Romans A. Association of microRNA expression with microsatellite instability status in colorectal adenocarcinoma. J Mol Diagn. 2010;12:433-440.

24. Bruno IG, Karam R, Huang L. Identification of a microRNA that activates gene expression by repressing nonsense-mediated RNA decay. Mol Cell. 2011;42:500-510.

25. Ortega-Molina A, Serrano M. PTEN in cancer, metabolism, and aging. Trends Endocrinol Metab. 2013;24:184-189.

26. Carracedo A, Alimonti A, Pandolfi PP. PTEN level in tumor suppression: how much is too little? Cancer Res. 2011;71:629-633.

27. Gao Y, Luo LH, Li S, Yang C. miR-17 inhibitor suppressed osteosarcoma tumor growth and metastasis via increasing PTEN expression. Biochem Biophys Res Commun. 2014;444:230-234. 


\section{Publish your work in this journal}

OncoTargets and Therapy is an international, peer-reviewed, open access journal focusing on the pathological basis of all cancers, potential targets for therapy and treatment protocols employed to improve the management of cancer patients. The journal also focuses on the impact of management programs and new therapeutic agents and protocols on patient perspectives such as quality of life, adherence and satisfaction. The manuscript management system is completely online and includes a very quick and fair peer-review system, which is all easy to use. Visit http://www.dovepress.com/testimonials.php to read real quotes from published authors.

Submit your manuscript here: http://www.dovepress.com/oncotargets-and-therapy-journal 